nitrate solution, and volumetrically on the same sample by determining the excess of silver in the filtrate with thiocyanate.

Calc. for $\mathrm{C}_{3} \mathrm{H}_{9} \mathrm{PSe}$ : Se, 50.99. Found: gravimetrically, 51.11, 51.21; volumetrically, 50.64, 50.76 .

The phosphorus analogue of choline has been prepared. This and other similar compounds of phosphorus, arsenic and antimony will be described in papers to be published later.

NEW YORK UNIVERSTY, NEW YORE, N. Y.

[Contribution from the Chemical laboratory of Tufts College.]

\title{
THE ADDITION OF AROMATIC AMINES TO BROMONITRO- STYRENE.
}

BY David E. WORRaLL.

Received January 22, 1921.

\section{Introduction and Theoretical.}

The $\alpha-\beta$ unsaturated nitro compounds contain a conjugate unsaturated system which makes their addition reactions of particular interest. The easiest and most convenient to prepare in quantity is nitro-styrene, $\mathrm{C}_{6} \mathrm{H}_{5} \mathrm{CH}=\mathrm{CHNO}_{2}$. Hence this compound was first chosen in an investigation of the addition of ammonia and substituted ammonias to unsaturated nitro compounds. ${ }^{1}$ The sensitiveness of nitro-styrene, particularly the ease with which it polymerizes was found to restrict the scope of the reaction to those additions which took place readily. Those reactions in part have been reinvestigated with the bromo derivative of nitrostyrene, a substance which does not polymerize, and the limits more clearly defined.

Bromonitro-styrene was first prepared by Priebs, ${ }^{2}$ who assigned to it the structure $\mathrm{C}_{6} \mathrm{H}_{5} \mathrm{CBr}=\mathrm{CHNO}_{2}$ because of its solubility in aqueous alkali. Thiele ${ }^{3}$ later showed that the solubility in alkali is due to the iormation of a salt through addition. He proposed $\mathrm{C}_{6} \mathrm{H}_{5} \mathrm{CH}=\mathrm{CBrNO}_{2}$ as the correct formula, based on the behavior of the potassium methylate addition product of bromonitro-styrene which reacts with bromine to form a compound that is insoluble in alkali.

A much more satisfactory proof of the correctness of Thiele's formula has been found in this research. One of the decomposition products resulting from the action of organic bases on bromonitro-styrene is bromonitro-methane, showing that bromine and the nitro group are held by the same carbon atom.

The first step in the reaction between bromonitro-styrene and organic

${ }_{1}^{1}$ Dissertation, Harvard University. This work was done under the direction of Professor Michael.

2 Priebs, Ann., 225, 343 (1884).

3 Thiele and Haeckel, ibid., 325, 2 (1902). 
bases is direct addition and under favorable conditions the addition product can be isolated. With $p$-toluidine the conditions are particularly favorable for the toluidino derivative can be prepared by mixing molecular quantities of the two substances in alcohol without any precautions and even by warming in the absence of a solvent, although in this case the product is much less pure. The speed of the reaction is so great that it is possible to have the solid addition product form in less than a minute after mixing warm $p$-toluidine and bromonitro-styrene. The toluidino derivative is decomposed by heat into bromonitro-methane and benzaltoluidine. It is also decomposed by hydrochloric acid into benzaldehyde, bromonitro-methane and toluidine. The first step is the formation of a double salt, which can be isolated by using dry hydrogen chloride and anhydrous ether. The salt breaks up into the hydrochloride of toluidine. and bromonitro-styrene, followed by hydrolysis of the latter substance.

$$
\begin{gathered}
\mathrm{C}_{6} \mathrm{H}_{5} \mathrm{CH}\left(\mathrm{NHC}_{6} \mathrm{H}_{4} \mathrm{CH}_{3}\right) \mathrm{CHBrNO}_{2}+\mathrm{HCl} \longrightarrow \mathrm{C}_{6} \mathrm{H}_{5} \mathrm{CH}\left(\mathrm{NH}_{2} \mathrm{ClC}_{6} \mathrm{H}_{4} \mathrm{CH}_{3}\right)- \\
\mathrm{CHBrNO}_{2} \longrightarrow \mathrm{CH}_{3} \mathrm{C}_{6} \mathrm{H}_{4} \mathrm{NH}_{2} \mathrm{HCl}+\mathrm{C}_{6} \mathrm{H}_{5} \mathrm{CH}=\mathrm{CBrNO}_{2}- \\
\mathrm{C}_{6} \mathrm{H}_{5} \mathrm{CH}=\mathrm{CBrNO}_{2}+\mathrm{H}_{2} \mathrm{O} \longrightarrow \mathrm{C}_{6} \mathrm{H}_{5} \mathrm{CHO}+\mathrm{CH}_{2} \mathrm{BrNO}_{2} .
\end{gathered}
$$

Bromonitro-phenyl-toluidino-ethane is soluble in aqueous alkali, forming a metal salt from which the original substance is regenerated on careful treatment with acid. This proves the presence of hydrogen $\alpha$ to the nitro group; it also proves that the toluidino group adds to the $\beta$ carbon (to $\mathrm{NO}_{2}$ ) in the addition of toluidine to bromonitro-styrene.

The speed of reaction of phenylhydrazine with bromonitro-styrene is sufficient to permit control of conditions and by use of the proper solvent and low temperatures, it is possible to isolate the hydrazino derivative, $\mathrm{C}_{6} \mathrm{H}_{5} \mathrm{CH}\left(\mathrm{NHNHC}_{6} \mathrm{H}_{5}\right) \cdot \mathrm{CHBrNO}_{2}$. It is easily decomposed by acids, bases or heat into the phenylhydrazone of benzaldehyde and bromonitromethane.

The addition of the organic base usually takes place much more slowly: Such is the case with aniline. The first step in the reaction may be regarded as $1-4$ addition to the conjugate system.

$$
\mathrm{C}_{6} \mathrm{H}_{5} \mathrm{CH}=\mathrm{CBrNO}_{2}+\mathrm{C}_{6} \mathrm{H}_{5} \mathrm{NH}_{2} \rightarrow \mathrm{C}_{6} \mathrm{H}_{5} \mathrm{CH}\left(\mathrm{NHC}_{6} \mathrm{H}_{5}\right) \mathrm{BrC}=\mathrm{N}^{-\mathrm{O}} \mathrm{H}
$$

'This compound under favorable conditions immediately rearranges.

$\mathrm{C}_{6} \mathrm{H}_{5} \mathrm{CH}\left(\mathrm{NHC}_{6} \mathrm{H}_{5}\right) \mathrm{BrC}=\mathrm{N} \stackrel{\mathrm{O}}{\mathrm{O}}-\mathrm{H} \rightarrow \mathrm{C}_{6} \mathrm{H}_{5} \mathrm{CH}\left(\mathrm{NHC}_{6} \mathrm{H}_{6}\right) \mathrm{CHBrNO}_{2}$. Since the speed of the first reaction is slow the new substance is in contact with an excess of the free base which decomposes it into bromonitromethane and benzal-aniline.

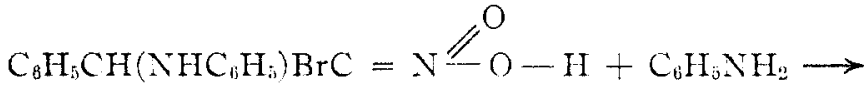

$$
\begin{aligned}
& \mathrm{C}_{6} \mathrm{H}_{4} \mathrm{CH}=\mathrm{NC}_{6} \mathrm{H}_{4}+\mathrm{CH}_{2} \mathrm{BrNO}_{2}+\mathrm{C}_{6 j} \mathrm{H}_{6} \mathrm{NH}_{2} \text {. }
\end{aligned}
$$


The carbon chain is weakened by the accumulation of negative groups on the terminal carbon atom so that its rupture is easily brought about at this point. The Schiff base is decomposed by bromonitro-methane if water is present and because of the marked hydroscopicity of aniline this decomposition is difficult to avoid. Hence the final product is a mixture of benzaldehyde, bromonitro-methane and aniline, which on long standing undergoes further changes evidenced by gas formation and the odor of isonitrile. Attempts were made to avoid an excess of base and to speed up the initial reaction by dropwise addition of aniline to the molecular equivalent of bromonitro-styrene at somewhat elevated temperatures. Decomposition into benzal-aniline and bromonitro-methane took place under these conditions, for the initial product is decomposed by heat as well as by bases. Bromonitro-methane is easily detected even when the concentration is low because of the powerful lachrymatory properties of its vapor. Therefore, since its presence necessarily depends on the previous formation of the addition compound it serves as a delicate and extremely convenient indicator.

\section{Experimental.}

a-Bromonitro- $\beta$-phenyl- $p$-toluidino-ethane, $\mathrm{C}_{6} \mathrm{H}_{5} \mathrm{CH}\left(\mathrm{NHC}_{6} \mathrm{H}_{4} \mathrm{CH}_{3}\right) \mathrm{CHBrNO}_{2}$ To $20 \mathrm{~g}$. of finely powdered bromonitro-styrene suspended in $35 \mathrm{cc}$. of alcohol and cooled by ice-water, was added dropwise with stirring the molecular equivalent $(9.2 \mathrm{~g}$.) of $p$-toluidine dissolved in $15 \mathrm{cc}$. of warm alcohol. An immediate reaction took place, the solution becoming orange in color; at the same time the bromonitro-styrene for the most part went into solution. Crystal formation started in several minutes and quickly spread through the mixture, forming a thick magma. The product which was filtered off and dried after standing an hour weighed approximately $20 \mathrm{~g}$. and melted at $103^{\circ}$ to $104^{\circ}$. Nearly $7 \mathrm{~g}$. of a less pure product, melting at $101^{\circ}$ to $103^{\circ}$, separated from the mother liquor on allowing the latter to evaporate overnight at room temperature to a small bulk. The first crop was purified by recrystallization from warm alcohol and dried a.t room temperature.

Analysis. Calc. for $\mathrm{C}_{15} \mathrm{H}_{15} \mathrm{O}_{2} \mathrm{~N}_{2} \mathrm{Br}$ : $\mathrm{Br}, 23.9$. Found: 23.4 .

The new substance is easily dissolved by ether, benzene and warm alcohol, but only sparingly soluble in cold alcohol and nearly insoluble in petroleum ether. It may be crystallized from benzene-petroleum-ether mixture or better from alcohol. If the liatter solvent is used, because of the ease with which the toluidino derivative is decomposed by heat into other substances, it is best to work with not too large quantities and to use moderate temperatures. A satisfactory procedure is to dissolve several grams of the compound in the minimum amount of warm $\left(40-50^{\circ}\right)$ alcohol, allow the nixture to stand at room temperature until crystal formation has started, then cool in ice-water and immediately filter by suction. It crystallizes from alcohol in narrow gold-yellow plates that melt with foaming at $107-108^{\circ}$ if the usual rate of heating is followed. The melting point is not constant and varies markedly according to the rate of heating. If heated rapidly it melts as high as $110^{\circ}$ to $111^{\circ}$, while on the other hand it s.lowly changes to a liquid when heated on a steam-plate for an hour.

The toluidino derivative is a sensitive substance characterized by the ease with which bromonitro-methane splits off under the influence of heat. It is also broken down by acids and bases, easily oxidized and acted upon by halogens and by nitric acid. Hence it. is difficult to obtain a pure preparation. The original crystals in all cases contain 
traces of bromonitro-methane, which are not entirely removed by subsequent recrystallizations, although the odor of bromonitro-methane disappears from the dry product on standing. The addition compound may be obtained by mixing the components at room temperature or even by warming, but the product obtained is much less pure than one in which the precautions previously mentioned are observed and which is sufficiently pure for most purposes.

The Action of Water.-Five $g$. of the substance was heated with several hundred g. of water in a distilling flask. It quickiy melted and darkened in color. A small amount of a nearly colorless oil distilled over mixed with water. An excess of alkali was added and the mixture shaken out with ether. The aqueous layer was separated, neutralized with acid and the oil liberated identified as bromonitro-styrene. The ether extract on evaporation left behind a few drops of a dark colored oil having a pronounced odor of toluidine. It was separated into two parts, one of which was treated with phenylhydrazine, the resulting crystals being dried on filter paper and recrystallized from alcohol. The melting point $154^{\circ}$ to $155^{\circ}$ identified the substance as the phenylhydrazone of benzaldehyde. The other portion of the original oil formed a pasty mass with hydrochloric acid which was dried on a porous plate, washed with ether and finally mixed with sodium hydroxide. The resulting oil partially solidified on cooling and proved to be impure toluidine, but the amount was insufficient for further manipulation. The major part of the original substance remained in the flask during the distillation and formed a black vitreous lump.

Action of Hydrochloric Acid.-The toluidino derivative by heating with conc. hydrochloric acid was quickly decomposed into benzaldehyde, bromonitro-methane and $p$-toluidine hydrochloride in addition to considerable tarry material. To $3 \mathrm{~g}$. of the toluidine addition product dissolved in dry ether hydrogen chloride was added with occasional shaking. The color of the solution rapidly faded until it was nearly colorless. A voluminous precipitate of feathery white needles formed which were purified by repeated decantation with dry ether. The new substance is the hydrochloric acid salt of the toluidino compound melting with decomposition at $102^{\circ}$ to $104^{\circ}$. It is very sensitive to moisture regenerating the toluidino compound and hydrochloric acid. A similar reaction takes place with ordinary alcohol.

Action of Sodium Hydroxide.-Five g. of pulverized bromonitro-phenyl-toluidinoethane was suspended in a small volume of $20 \%$ sodium hydroxide solution for several hours during which time it gradually changed to a thick white paste of microscopic needles. The mass was suction-filtered, washed with cold water and dried on a porous plate. It was recrystallized from benzene.

Analysis. Subs., 0.320: $\mathrm{Na}_{2} \mathrm{SO}_{4}, 0.0581$. Calc. for $\mathrm{C}_{16} \mathrm{H}_{14} \mathrm{O}_{2} \mathrm{~N}_{2} \mathrm{BrNa}: \mathrm{Na}$, 6.4. Found: 5.9 .

The sodium derivative is sparingly soluble in cold water and in benzene from which it may be crystallized in feathery needles.

The sodium salt was also prepared by heating the toluidino derivative with sodium hydroxide solution. The color gradually faded as the substance went into solution. An oil separated out which hardened on cooling to a white crystalline mass. On longer heating decomposition took place so the mixture was then steam distilled. A few drops of a clear oil first came over, possessing the characteristic nauseating odor of isonitrile. This was followed by more oily drops, pale yellow in color, which were identified through the aid of phenyl hydrazine as benzaldehyde. The residue from the steam distillation contained some solid material which was filtered off and examined later. The filtrate on acidification gave no indication of the presence of bromonitromethane. Apparently this substance reacts with primary amines in the presence of alkali to form isocyanides. The solid residue was crystallized several times from al- 
cohol, separating finally as white plates which melted at $131^{\circ}$ to $132^{\circ}$ and were presumably hydrobenzoin.

Several grams of the sodium salt was dissolved in a large volume of water and cooled with ice. Carbon dioxide having no effect, an excess of dil. acetic acid was added slowly with stirring. The gold-yellow precipitate that settled out was separated, recrystallized from alcohol and identified by a mixed-melting-point determination as bromonitrophenyl-toluidino-ethane.

Action of Bromine.-Five $\mathrm{g}$. of the toluidino product was dissolved in chloroform, an excess of bromine added and the mixture refluxed for 30 minutes. Crystals soon formed amid copious evolution of hydrogen bromide. Altogether $2.8 \mathrm{~g}$. of the crystalline product was obtained from the mixture. It was easily purified by digestion with hot benzene in which it was insoluble. On filtering there resulted a mat of silky white needles that darkened on heating and decomposed at $210^{\circ}$ to $212^{\circ}$ with preliminary softening. The substance was found to be the hydrobromide of a low melting compound $\left(72-73^{\circ}\right)$ obtained by heating the salt with water followed by crystallization from alcohol diluted with water. It was eventually identified by a mixed-meltingpoint determination with a known sample of 3,5-dibromo-4-toluidine. ${ }^{1}$ On evaporation of the chloroform filtrate to a small bulk, crystals separated out which were washed with benzene and united with the first lot. The oily residue $(5.4 \mathrm{~g}$.$) contained$ bromonitro-methane, benzaldehyde and bromonitro-styrene.

Action of Nitric Acid.-Five g. of powdered bromonitro-phenyl-toluidino-ethane in small amounts was added with stirring to conc. nitric acid. It reacted instantly, partially going into solution. A dark tarry mass gradually accumulated in the acid, but on standing at room temperature, it eventually became crystalline. If the reaction was allowed to proceed too vigorously at first it foamed and liberated bromonitromethane. The acid mixture was then poured into water, filtered and thoroughly washed with warm alcohol in which the new compound is only slightly soluble. There remained approximately $4 \mathrm{~g}$. of pure substance. It crystallizes from hot alcohol in which it is sparingly soluble in flat pale yellow needles that melt at $154^{\circ}$ to $155^{\circ}$, with decomposition and foaming, due to the evolution of bromonitro-methane.

Analysis. Calc. for $\mathrm{C}_{15} \mathrm{H}_{13} \mathrm{O}_{6} \mathrm{~N}_{4} \mathrm{Br}$ : $\mathrm{Br}, 16.8$. Found: 16.4 .

The new substance is a dinitro derivative of bromonitro-phenyl-toluidino-ethane in which the nitro groups are located in the toluidino radical. This was determined by heating the nitro derivative with sodium hydroxide solution. Considerable tar formed but by steam distiliation benzaldehyde was separated and identified.

a-Bromonitro- $\beta$-phenylhydrazino-phenyl-ethane, $\mathrm{C}_{6} \mathrm{H}_{6} \mathrm{CH}\left(\mathrm{NHNHC}_{6} \mathrm{H}_{5}\right) \mathrm{CHBrNO}_{2}$ -Five g. of powdered bromonitro-styrene was suspended in $20 \mathrm{cc}$. of alcohol and cooled with ice-water. The molecular equivalent $(2.4 \mathrm{~g}$.) of freshly distilled phenylhydrazine dissolved in $10 \mathrm{cc}$. of alcohol was added slowly with stirring. The bromonitro-styrene for the most part went into solution quickly followed by a copious precipitate of small gold-yellow needles. The irritating odor of bromonitro-methane was apparent to a slight extent only. After standing about an hour in the ice-water the mixture was filtered and dried at room temperature. Yield about $4 \mathrm{~g}$. The hydrazino derivative was purified by dissolving in warm alcohol. The crystals separating out on cooling in ice-water were separated and dried. Melting point $83^{\circ}$ to $83.5^{\circ}$ with decomposition.

Analysis. Calc. for $\mathrm{C}_{14} \mathrm{H}_{14} \mathrm{O}_{2} \mathrm{~N}_{3} \mathrm{Br}$ : $\mathrm{Br}, 23.8$. Found: 23.2 .

The properties of the hydrazino compound are similar to those of bromonitro-

1 Wroblewski, Ann., r68, 188 (1877), who prepared this substance by the action of bromine vapor on $p$-toluidine hydrochloride, stated that it did not form salts with acids. 
phenyl-toluidino-ethane. It decolorizes potassium permanganate, reacts with bromine, forms a salt with hydrogen chloride. It is easily decomposed into benzal-phenylhydrazine and bromonitro-methane.

Five g. of bromonitro-styrene was refluxed for an hour with the molecular equivalent of phenylhydrazine and alcohol. The mixture was then poured into water, extracted with ether and evaporated to drynesss. The residue contained traces of ammonium bromide and phenylhydrazine hydrobromide. The ether extract was shaken out with sodium hydroxide solution, the aqueous layer acidified and again shaken with ether. A heavy oil $(2.6 \mathrm{~g}$.) remained after the evaporation most of which distilled at $146^{\circ}$ to $148^{\circ}$ and was bromonitro-methane. From the first ether extract was recovered $4.5 \mathrm{~g}$. of crude benzal-phenylhydrazine. The crude product was purified and identified with a known sample by the mixed-melting-point method.

The Action of Aniline.-To $5 \mathrm{~g}$. of bromonitro-styrene suspended in cold alcohol was added slowly with stirring the molecular equivalent of aniline. The alcohol became red in color while the styrene went into solution. The odor of bromonitro-methane was soon noted and complete decomposition into this substance, benzaldehyde and aniline was found to have taken place. Attempts to isolate the addition product were unsuccessful.

The Action of $o$ - and $m$-toluidines. - In both cases the products of the reaction with bromonitro-styrene were oils which contained bromonitro-methane.

The Action of $p$-Phenylene-diamine.-One $\mathrm{g}$. of the base was dissolved in alcohol and added to the equivalent amount of bromonitro-styrene suspended in alcohol. The odor of bromonitro-methane soon became apparent on standing, while a black pasty mass separated out. The tar was pressed on a porous plate and crystallized several times from alcohol, separating finally in irregular plates melting at $138^{\circ}$ to $140^{\circ}$. The substance was dibenzylidene-p-phenylene-diamine. It partially changed on longer standing with bromonitro-methane into the base and benzaldehyde.

The Action of Benzidine.-Molecular quantities of the two substances were mixed in alcohol solution. A heavy oil separated out on standing, but did not harden. It was washed with warm alcohol and crystallized from benzene in glistening plates melting at $231^{\circ}$ to $232^{\circ}$, which identified the substance as dibenzylidine benzidene.

The Action of $\beta$-Naphthylamine.-Molecular quantities of the base and the unsaturated compound were mixed in the customary manner. An oil separated out on long standing that was identified as a mixture of benzaldehyde, bromonitro-methane and naphthylamine.

The Action of Diphenylhydrazine.-Molecular amounts of the two substances were mixed in alcohol solution. As no apparent reaction took place the solution was warmed, resulting in oil formation. The oil hardened on standing to a solid which was recrystallized from alcohol in yellow crystals melting at $122^{\circ}$. Hence the diphenylhydrazone of benzaldehyde and bromonitro-methane were formed.

Action of Diphenylamine, etc.-Negative results were obtained with diphenylamine, $m$-nitro-aniline and dimethyl-aniline. Diphenylamine, for example, may be crystallized unchanged from warm bromonitro-styrene.

\section{Summary.}

Aromatic amines and hydrazines add readily to $\alpha$ - $\beta$ unsaturated nitro compounds containing halogen $\alpha$ to the nitro group.

Substituted aromatic amines containing negative groups do not react. 'The addition compounds as a rule decompose as fast as they form into 
bromonitro-methane and Schiff bases. The latter are decomposed by bromonitro-methane into benzaldehyde and amine.

The addition compounds of $p$-toluidine and phenylhydrazine may be isolated. They are amphoteric substances, forming salts with both metals and acids.

MEDFORD HILLSIDE, MASS.

[CONTRIBUTION FROM THE CHEMICAL LABORATORY OF THE UNIVERSITY OF ILLiNOIS.]

\section{THE APPLICATION OF VICTOR MEYER'S ESTERIFICATION LAW TO 2,6-XYLIC ACID AND ITS REDUCED DERIVATIVES. ${ }^{1}$}

By Ralph W. HuFFERd With William A. Noyes.

Received January 25, 1921.

\section{Historical.}

Victor Meyer found that aromatic acids with both ortho positions filled are not to be esterified by the usual method. The work of himself and his pupils ${ }^{2}$ led Meyer to formulate the rule which is known as Victor Meyer's Esterification Law. It states, "When in a substituted benzoic acid the two hydrogen atoms adjacent to the carboxyl group are substituted by such groups as $\mathrm{Br}, \mathrm{NO}_{2}, \mathrm{CH}_{3}$, etc., the acid is not esterifiable by alcohol and hydrochloric acid."

Meyer explained this difficulty of esterification by advancing the theory of steric hindrance. He believed that the groups adjacent to the carboxyl covered it up so as to prevent free collision between the alcohol molecule and the carboxyl group. A strong argument in favor of this explanation is the difference in behavior toward esterification of di-ortho-substituted benzoic acids and similarly substituted acids with the carboxyl removed from the ring by at least one atom. Symmetrical trimethyl-benzoic acid obeys the law but both mesityl-glyoxylic acid and mesityl-acetic acid esterify readily. In fact, even the ortho hydrogen atoms in benzoic acid are credited with some blanketing effect, since it was found that phenylacetic acid is more easily esterified than is benzoic acid itself.

Wegscheider ${ }^{3}$ advanced some data which are in accordance with Meyer's rule, but he objected to Meyer's steric hindrance explanation and declared that the effect is due to a state of tension within the molecule.

The most antagonistic critic of the steric hindrance idea is M. A. Rosan-

${ }^{1}$ An abstract of a thesis submitted by Ralph $W$. Hufferd in partial fulfilment of the requirements for the degree of Doctor of Philosophy in the Graduate School of the Lniversity of Illinois.

${ }^{2}$ Meyer, et. al., Ber. 27, 510, 1585, 3146 (1894); 28, 182, 1254, 2776, 3197, 3219 (1805); 29, 830, 839, 1397 (1896); Z. physik. Chem., 24, 219, 221 (1897).

'Wegscheider, Monatsch.f. Chem., r8, 643 (1897); Ber., 28, 2535 (1895). 